\title{
¿Es posible un tratamiento diferente de las adicciones? ${ }^{1}$
}

RESUMEN: Los tratamientos de los trastornos adictivos siguen la inercia de los criterios asistenciales de la segunda mitad del siglo XX. Aunque estos han dado una respuesta satisfactoria a ciertas personas, hay un numeroso grupo de alcohólicos y drogodependientes con repetidas experiencias terapéuticas fracasadas, expuestos en las recaídas a severos procesos de marginación. Estos hechos junto con las modernas hipótesis sustentadas en los resultados de las investigaciones y en las relaciones observadas entre drogas y trastornos mentales, animan a revisar los objetivos y medios del tratamiento de los drogodependientes.

PALABRAS CLAVE: Drogodependencias, tratamiento, análisis.
ABSTRACT: Drug addiction has today almost the same therapeutic approach that it had in the first sixties. The therapeutic models prevailing now a days have had success in some patients, although some others have repeted failures and relapses with margination problems associated. This facts plus the modern hypothesis about the drug dependence make necesary to review the goals and procedures in addictives disorders treatment.

KEY WORDS: Drug addiction, therapeutic, review

\section{Introducción}

Desde hace muchos años se habla en España de las drogas, los toxicómanos y su tratamiento, y mientras se debate acerca de las ventajas e inconvenientes de la legalización, de la cuantía e idoneidad de las sanciones penales y administrativas a traficantes y consumidores, se anima a dar respuesta desde lo terapéutico a las graves consecuencias del consumo abusivo de drogas. A ello destinan cuantiosos recursos las administraciones públicas y numerosas organizaciones sociales: fundaciones, asociaciones, etc., alcanzando éxitos incuestionables. Sin embargo, las modernas hipótesis acerca del origen y mantenimiento del abuso y dependencia de drogas, sustentadas por los resultados de las investigaciones actuales del cerebro y el genoma humano y en las relaciones empíricas y epidemiológicas entre drogas y trastornos mentales (1), permiten afirmar que es necesario revisar los objetivos y medios del tratamiento de drogodependientes porque se han quedado anticuados.

La intervención terapéutica en drogodependencias se ha sustentado en las ideas higienistas de principios del siglo $\mathrm{XX}$, los prejuicios acerca de las drogas y la presión

Rev. Asoc. Esp. Neuropsiq., 2001, vol XXI, n. ${ }^{\circ} 79$, pp. 9-53

${ }^{1}$ Agradecimiento: A todos los compañeros, colegas y amigos que con la lectura y comentarios de este texto han contribuido a enriquecerlo. 
social, la influencia del psicoanálisis y en muchas ocasiones ha quedado a expensas de criterios políticos o de quienes aseguraban estar en posesión de la receta para resolver el problema de la mayoría.

\section{Nuevos problemas para una sociedad en cambio}

Cuando los problemas derivados del consumo de drogas ilegales, especialmente heroína, hacen eclosión en España en la década de los ochenta se configuran distintas percepciones sociales del toxicómano (2), con dos puntos de vista dominantes, por un lado quienes lo consideraban un "enfermo moral" y por otro quienes lo percibían como un "enfermo social", ambos con adscripciones ideológicas diferentes, la primera más vinculada al sector conservador y la segunda al progresista de una sociedad en plena transición de la dictadura franquista a la monarquía parlamentaria. Para quienes lo consideran un "enfermo moral", el toxicómano es un vicioso, un degenerado que padece las consecuencias de un vicio irrefrenable, cuya curación, siempre difícil, pasa por el arrepentimiento y el proselitismo de la abstinencia. Los que lo ven como un "enfermo social" estiman que la dependencia y el abuso de drogas son una expresión del malestar de los jóvenes ante una sociedad consumista, volcada en el individualismo, que brinda muy pocas oportunidades a los más desfavorecidos y que no ofrece alternativas ni alicientes. La curación requiere de la movilización social, de la participación ciudadana para conseguir un entorno más humanizado que además proporcione comprensión, apoyo y acompañamiento al drogodependiente en su proceso de recuperación.

En el ámbito profesional, cuando a principios de los ochenta muchos heroinómanos necesitan y piden tratamiento, el modelo tradicional de asistencia psiquiátrica, que giraba alrededor de los manicomios, está en crisis. Se están llevando a cabo los cambios propiciados por la denominada reforma psiquiátrica, que desplaza progresivamente, y con distintos ritmos en la geografia nacional, la asistencia del enfermo mental de los hospitales psiquiátricos a otros recursos asentados en la comunidad e integrados en las redes sanitarias generales: centros de salud mental, unidades de hospitalización psiquiátrica, centros de día, hogares tutelados, etc. Con los hospitales psiquiátricos se cierran los lugares habituales de aislamiento de alcohólicos y otros toxicómanos desde la promulgación de la Ley de julio de 1931 (3), "dictando reglas relativas a la asistencia a enfermos psíquicos", y por tanto el principal referente de experiencia asistencial de este tipo de problemas. La psiquiatría española de vanguardia está volcada en el empeño de organizar un marco asistencial alternativo más respetuoso con los derechos de los pacientes y en reintegrar a las personas olvidadas en los psiquiátricos en su medio socio-familiar. La tarea es ardua, pero hay un decidido compromiso de poner en marcha un modelo de atención psiquiátrica inspirado en la psiquiatría alternativa y forjado en años de oposición a un modelo obsoleto. En el esfuerzo queda poco tiempo para la reflexión y el análisis de los nuevos problemas de salud mental que surgen en el seno de una sociedad sometida a los cambios acelerados de la transición política. En consecuencia, cuando el problema de las drogas se expresa con virulencia la corriente psiquiátrica preponderante está mirando a otro lugar. 


\section{Nace una nueva red de tratamiento}

Las peticiones de tratamiento relacionadas con las drogas desbordan por su número y cualidad a la red de recursos socio-sanitarios y empujan con la alarma social desencadenada por las dramáticas consecuencia personales, familiares y sociales de la drogodependencia, a las administraciones públicas a desarrollar la respuesta al problema. Alrededor del Plan Nacional y de los Planes Autonómicos sobre Drogas se crea una red asistencial, con diferencias organizativas, pero eminentemente específica, es decir en paralelo y no integrada en las estructuras asistenciales existentes. Aparecen y se multiplican el número de centros ambulatorios, unidades de desintoxicación hospitalaria, comunidades terapéuticas y centros de día dedicados en exclusiva al tratamiento de toxicómanos con un objetivo principal, la abstinencia.

Los programas de tratamiento no son ajenos a los puntos de vista de las percepciones sociales dominantes $y$, por lo general, consideran que los pacientes deben hacer un esfuerzo por integrarse en las actividades y adaptarse a las normas del tratamiento. Como en el caso de los alcohólicos, el tratamiento de los adictos a drogas “..., parece cosa de justicia. El bebedor, como el delincuente, tiene que reconocer los hechos ante todo: falta confesada, medio perdonada. Al confesarse culpable, dispuesto a enmendarse, ya casi está en el buen camino, el camino de los que sólo desean su bien, de los que saben que es lo mejor para él" (Roger Gentis) (4). Apenas se presta atención a las circunstancias personales y sociales que impide a los pacientes acercarse al listón de la abstinencia o mantenerse en él, tales como la comorbilidad psiquiátrica y el efecto de los años de consumo sobre la situación social y familiar, la salud mental y física y, en consecuencia, sobre la disposición y capacidad de cambio. La "falta de motivación" del paciente o el que "no hubiera tocado fondo" eran los argumentos más generalizados y habituales para justificar los numerosos abandonos y recaídas. Lamentablemente, la recaída del toxicómano conduce a la calle, sin refugio posible en el bar, como el alcohólico, sumando al deterioro de la autoestima, la salud, y la familia, el de la exclusión social y la delincuencia.

La aplicación de estos modelos de tratamiento mientras se desarrolla una asistencia psiquiátrica comunitaria, contribuyó a modificar radicalmente la imagen social del marginado, pasando de estar configurada alrededor del "loco" y el alcohólico, al toxicómano. En efecto, los tratamientos farmacológicos cada vez más eficaces para algunos de los trastornos mentales más graves, en especial la esquizofrenia, unido a la atenuación de los procesos de marginación asociados a la enfermedad mental que proporcionaron los programas de rehabilitación e integración, consiguieron normalizar notablemente la vida de estos pacientes. Es cierto que hay personas con estos trastornos que entran en circulos de exclusión y cronicidad, pero también lo es que hay recursos asistenciales de calidad donde pueden ser acogidos y tratados, recursos potencialmente capaces de adecuar las prestaciones a las necesidades de los usuarios. 
Por el contrario, la farmacopea para el tratamiento de los drogodependientes es básicamente la misma desde hace más de veinte años y desde entonces se ha aplicado con idénticos criterios: tratamientos de sustitución con metadona o de antagonización con naltrexona con psicoterapia de apoyo para abandonar el consumo de drogas. Al igual que otros programas psico-sociales: comunidades terapéuticas, centros de día, etc., han estado a disposición de los toxicómanos animados a dejar las drogas, olvidando a quienes mantenían su uso. Como la dependencia de drogas es un itinerario jalonado de periodos de abstinencia y de consumo y ningún programa de tratamiento garantiza los resultados, se ha ido constituyendo un grupo de drogodependientes con numerosas experiencias terapéuticas fracasadas, expuestos en las recaídas a severos procesos de marginación, que se han convertido en el paradigma de las dificultades relacionadas con las drogas y provocan un fortísimo rechazo social. Son personas desmoralizadas, sin fuerza para el cambio, deterioradas fisica y socialmente, sin lazos familiares ni red social y con graves trastornos y enfermedades mentales asociadas, usuarios incómodos de los recursos sociosanitarios, que deambulan por las ciudades y se concentran alrededor de establecimientos para poblaciones marginales o de los puntos de venta de drogas.

\section{Cambia el enfoque de los tratamientos}

A finales de los ochenta y principios de los noventa la altísima prevalencia del sida en toxicómanos y la necesidad de contener su difusión introdujo un cambio sustancial en el enfoque de los tratamientos, propiciando la aceptación generalizada de la reducción de los daños derivados del consumo como un objetivo más de la intervención terapéutica. Este cambio permitió desplazar el foco de atención en la asistencia de la abstinencia al sujeto y así descubrir a personas con problemas y una vivencia de los mismos que requería ser escuchada (5).

Paralelamente, la investigación de la comorbilidad psiquiátrica en usuarios de drogas desveló la importancia de los trastornos duales en el origen y mantenimiento de la conducta adictiva (6). Asimismo, el estudio de los procesos individuales de cambio para el abandono del consumo de drogas, identificó distintas etapas, desde la precontemplación a la actuación y el mantenimiento, que implican actitudes diferentes del consumidor y que requieren intervenciones distintas del terapeuta (7). En suma, se dieron pasos fundamentales para contemplar al consumidor de drogas como un sujeto con un trastorno intimamente relacionado con la enfermedad mêntal que pasa por momentos distintos en relación a su capacidad y deseo de cambio.

Esta nueva perspectiva descubre al toxicómano como un enfermo con un grave problema invalidante, la dependencia, que ha encontrado en las drogas la esencia, el alivio de las frustraciones, las ansiedades, la agresividad, la tristeza, las vivencias despersonalizadoras, y otras causas de malestar subjetivo. De este modo, el adolescente con trastornos del comportamiento, la persona retraida, depresiva, con dificultades para contener la impulsividad o las fobias, etc., encuentran el bálsamo reparador de sus 
molestias. Las usan hasta la dependencia y entonces cuando quieren recuperar el control de la relación con la droga se dan cuenta que no pueden. Los cambios neurofisilógicos, la desestabilización del precario bienestar psíquico, la rebaja de la escasa autoestima por la dependencia y las crisis familiares, y la sustitución de la red social normalizada por un grupo de pares al que le unen fuertes vínculos, llevan a la recaída en el uso incontrolado cuando lo que se busca desesperadamente es recuperar la convivencia armoniosa con la sustancia.

Por tanto, no se trata sólo de viciosos, ni de jóvenes sin oportunidades, sino de enfermos cuyo tratamiento necesita de una indicación terapéutica adecuada, sustentada en la exploración psicopatológica, en la identificación del momento del paciente en el ciclo de cambio y en la petición de ayuda desde un contexto determinado. Para esto, es imprescindible la escucha atenta de la vivencia del problema, libre de apriorismos y prejuicios, dejando fuera de lugar el "todo vale para todos". El drogodependiente tiene derecho a recibir un tratamiento digno, acorde a sus características y que tenga en cuenta lo que el conocimiento científico ha permitido saber de su enfermedad.

\section{Hacia un renovado modelo asistencial}

Cada vez con más fuerza, en los foros de debate, en los lugares de encuentro y discusión de profesionales y personas interesadas en el tema, en los documentos de conclusiones y recomendaciones de reuniones científicas y seminarios (8), se pone de manifiesto que ha llegado el momento de mantener una actitud nueva ante el tratamiento de los trastornos por uso de drogas que tenga en cuenta dichos criterios, porque con una asistencia adecuada se puede evitar que los más jóvenes se cronifiquen, aliviar la marginación de los crónicos, disminuir las tensiones sociales de la convivencia con eștos colectivos y modificar la percepción social del toxicómano.

Se ha de tener en cuenta que hoy en día conviven dos perfiles marcadamente diferentes de usuarios de drogas con problemas y demandas de tratamiento muy distintas. Por un lado el heroinómano crónico, con una larga historia de consumo, múltiples tratamientos y numerosos problemas legales, multidemandante, que hace uso de numerosos recursos sociales y sanitarios, en especial, de los de más fácil acceso a poblaciones marginales. De otro lado, jóvenes consumidores de alcohol u otras drogas, cuya frecuencia de uso va desde el abuso al exceso, que llegan al tratamiento generalmente empujados por la familia con síntomas de dependencia o de algún trastorno mental o del comportamiento donde el consumo de sustancias se entremezcla en la crisis propia del crecimiento y desarrollo. Son personas que acuden a las redes normalizadas de tratamiento y no identifican sus dificultades con las de un adicto. Su vinculación a los centros públicos de tratamiento es elástica (aparecen y desaparecen), díficil de incardinar al sistema tradicional de atención ambulatoria y constituyen un grupo con notables riesgos de deterioro y cronificación. 
Para atender a esta realidad variada y compleja, es necesario innovar la conceptualización y organización de la asistencia a los trastornos por uso de drogas, observando los siguientes principios:

1. Todo drogodependiente tiene derecho a asistencia, a recibir toda la ayuda que sea posible cuando sea preciso.

2. La asistencia, el tratamiento y las intervenciones de apoyo terapéutico deben realizarse desde la corresponsabilidad, eligiendo para cada caso el abordaje más apropiado, teniendo en cuenta la opinión del toxicómano acerca de su problema y la manera de resolverlo, así como los condicionantes involuntarios, el contexto y el momento del paciente en el ciclo de cambio.

3. La corresponsabilidad supone que el tratamiento debe contar con la libertad y el compromiso del sujeto, salvo circunstancias que lo impidan, e irá dirigido a alcanzar los objetivos acordados con el paciente.

4. Los objetivos de la asistencia irán encaminados a mejorar la salud y calidad de vida del paciente, siendo atendido y tratado con arreglo a las mismas normas aplicadas a los demás enfermos.

Para trabajar con estos principios es necesario reorganizar la red de recursos de asistencia, poniendo en juego los medios necesarios para convertirla en un red destinada a estabilizar, prestar apoyo en los procesos de recuperación y rehabilitación y proporcionar soporte en las recaídas. Una red flexible, reutilizable, que aproveche los recursos existentes, que tenga en cuenta los costes sociales de su presencia y con capacidad de dar respuesta a las manifestaciones cambiantes del problema. En definitiva una red con las siguientes características:

1. Normalizada, de manera que el consumo problemático de drogas sea visto como una expresión más del malestar del sujeto en su contexto biopsicosocial y los adictos puedan recibir asistencia en los mismos lugares que el resto de los ciudadanos con problemas o necesidades de similar naturaleza, sin detrimento de la existencia de lugares específicos para determinadas actividades o la atención a personas con problemas especiales.

2. Profesionalizada. El tratamiento de los trastornos por uso de drogas requiere de una formación y entrenamiento pormenorizádo en los ámbitos de la medicina y la psicopatología de las drogas, por tanto las indicaciones terapéuticas deben ser hechas exclusivamente por profesionales titulados y formados en estos ámbitos, responsables últimos de las consecuencias de sus decisiones ante el paciente, su familia y la sociedad. En esta línea, la Comisión Nacional de Psiquiatría en el informe de 1997 reclama el campo clínico del alcoholismo y otras drogodependencias como propio de la psiquiatría (9).

3. Suficiente, es decir, capaz de dar la atención precisa al paciente que lo necesite y de adaptarse a las necesidades de los pacientes, y no a la inversa. 
4. Complementaria, que rompa de manera efectiva con la dicotomía entre programas libres de drogas o de abstinencia y programas de reducción del daño, de modo tal que el criterio de acceso a los recursos sea conseguir la mejora de la salud y de la calidad de vida, así como contribuir a que la persona desarrolle recursos personales y sociales para ello.

5. Respetuosa con el entorno. Aunque el desarrollo de una red con los criterios que se señalan mejorará notablemente la ațención a las personas con problemas por uso de drogas y disminuirá las tensiones sociales relacionadas con este colectivo, seguirá habiendo personas gravemente afectadas necesitadas de asistencia. Su tratamiento habrá de tener en cuenta el impacto sobre el entorno y terceras partes afectadas para atenuarlos en la medida de lo posible. Así, se deberá evitar concentrar a los enfermos más graves en un mismo lugar, buscar espacios para la atención próximos a centros sociosanitarios y de servicios, alejados de las áreas eminentemente residenciales, y promover equipamientos en estas zonas como mecanismo compensador. 


\section{BIBLIOGRAFÍA}

1. Kaplan,H.I.; Sadock,B.J., 2000. Sinopsis de Psiquiatria, $8^{\mathrm{a}}$ Edición española, pp. 426-444. Editorial Médica Panamericana, Madrid.

2. Rodriguez Cabrero,G., 1993, "Drogodependencias y exclusión social desde la reflexión sociológica", en Las drogodependencias: perspectivas sociológicas actuales, Ilustre Colegio Nacional de Doctores y Licenciados en Ciencias Políticas y Sociologia, Madrid.

3. Aparicio,V.; Sánchez,A., 1997, "Norma y ley en la psiquiatría española", Revista de la Asociación Española de Neuropsiquiatria, vol. XVII, 61, pp. 125-145.

4. González Duro,E., 1998, "Psiquiatría versus alcoholismo", Revista de la Asociación Española de Neuropsiquiatria, vol. XVII, 66, pp. 361-372.

5. Marina,P., 1999, “¿Qué nos han enseñado los estudios de seguimiento de toxicómanos?", Adicciones, vol. 11, 3, pp. 237-241.

6. Rounsaville,B.J.; Weissmann,M.M.; Kleber,H.; Wilber,C., 1982, "Heterogeneity of psychiatric diagnosis in treated opiate addicts", Archives of General Psychiatry, 39, pp. 161-166.

7. Prochaska,J.O.; DiClemente,C.; 1982, "Transtheoretical therapy: toward a more integrative model of change", Psychoterapy: Theory, Research and Practice, 19, pp. 276-278.

8. Seminario Interdisciplinar de Expertos, 1998, Aspectos Éticos de los Programas de Sustitución para Dependientes a Opiáceos en España. Conclusiones y recomendaciones. Fundación de Ayuda contra la Drogadicción y Fundación de Ciencias de la Salud (Instituto de Bioética), Madrid, 11 páginas.

9. Comisión Nacional de la Especialidad de Psiquiatría (CNEP), 1998, "Documento de la CNEP de 24 de junio de 1997", Revista de la Asociación Española de Neuropsiquiatria, vol. XVIII, 68, pp. 749-758.

Fecha de recepción: 12/2/01

* Doctor en Medicina

LUGAR DE TRABAJO:

Unidad de Tratamiento Toxicomanías de Oviedo :

Servicios de Salud Mental del Principado de Asturias

Hospital Central de Asturias

C/ Julián Clavería s/n - 33006 Oviedo

CORRESPONDENCIAA:

Pedro A. Marina González

$\mathrm{C}$ / Miguel de Unamuno $19,8^{\circ} \mathrm{A}$

33010 Oviedo - E-mail: pepaz arrakis.es 